\title{
Aspectos pragmáticos y estratégicos del desarrollo de la destreza de expresión escrita en Filología Española en la tercera década del siglo XXI
}

\section{Pragmatic and strategic aspects of the development of written expression skills in Spanish Philology in the third decade of the XXI century}

\author{
Monika Grabowska \\ Uniwersytet Wrocławski (Polonia) \\ monika.grabowska@uwr.edu.pl \\ Magdalena Krzyżostaniak \\ Uniwersytet Wrocławski (Polonia) \\ magdalena.krzyzostaniak@uwr.edu.pl
}

\begin{abstract}
Written expression is one of the most important skills in philological studies. Subjected to a constant formative and summative evaluation, it plays a distinctive role and shows both the achievements and the shortcomings of the student's linguistic, sociolinguistic and pragmatic skills. In this analysis, we would like to focus on this last component of communicative competence, but also on the strategies of the learners connected with their practice of various forms of writing in the academic and private sphere. The paper summarizes the results of a survey completed by Spanish philology students from the Department of Romance Philology at the University of Wroclaw during the summer term of 2018/2019 academic year. One of the objectives of the survey was to find out to what extent the PNJH Pisanie program corresponds to the needs and expectations of current students in Poland.
\end{abstract}

Keywords: pragmatic competence, strategic competence, writing skills, Spanish studies 


\section{OBJETO, PROPÓSITO Y METODOLOGÍA DE LA INVESTIGACIÓN}

La expresión escrita es, por excelencia, una de las destrezas más importantes que se debe desarrollar durante los estudios filológicos. Está sometida a una constante evaluación formativa y sumativa. Se practica en cada cuatrimestre y no se limita a las clases de instrucción lingüística, de las que la producción escrita forma parte. Esta destreza desempeña un papel distintivo (los filólogos pertenecen a una minoría social con competencias suficientes como para cumplir con los requisitos del nivel C1 y C2 del Marco Común Europeo de Referencia para las Lenguas), evidenciando tanto los logros, como las faltas de las competencias lingüística, sociolingüística y pragmática del estudiante.

En este análisis queremos enfocarnos ante todo en ese último componente de la competencia comunicativa, entendida, según el MCER (2002), como "el conocimiento que posee el usuario o alumno de los principios según los cuales los mensajes se organizan, se estructuran y se ordenan", y "se utilizan para realizar funciones comunicativas", secuenciándose "según esquemas de interacción y de transacción" (p. 120)1.

En los albores de la tercera década del siglo XXI se está observando una creciente disparidad entre los retos que pueden plantear a los estudiantes géneros y formas pertenecientes a la alfabetización académica (ing. academic literacy) y las nuevas alfabetizaciones (ing. 21st century literacy) a las que la generación de nativos digitales (Prensky, 2001) se dedica con una intensidad difícil de comprender por los inmigrantes digitales (Prensky, 2001). El concepto de las nuevas alfabetizaciones no se limita a la producción de textos escritos sino que implica todo un abanico de funciones pragmáticas ofrecido por los medios digitales en el espacio virtual junto con la multimodalidad, hipertextualidad y cooperación creativa (Hulin, Pelissier, 2012). En consecuencia, los estudiantes parecen estar dotados de competencias que les permiten crear textos escritos gracias a las nuevas tecnologías; sin embargo, las formas discursivas privilegiadas por dichas tecnologías (mensajes de texto, microblogs, chats, mensajes en foros etc.) no siempre se reconocen y no se les atribuye el mismo valor que a las formas del ámbito académico dado, entre otros, el carácter comunitario de las primeras.

En este contexto nos pareció importante indagar sobre las prácticas de expresión escrita emprendidas por los estudiantes dentro de su actividad extraacadémica, la importancia que atribuyen a la producción escrita en su futuro profesional y la evaluación del programa de instrucción lingüística que realizan. Se pretendió investigar hasta qué punto dicho programa de estudios responde a las necesidades de un estudiante que está a punto de entrar en el mercado laboral actual. Nos propusimos averiguar si las competencias contenidas en los programas de estudios facilitan que los estudiantes se adapten a los cambios (cognitivos, según la descripción de Prensky, 2001; o sociales, según Harari, 2018) del mundo que los rodea, incluyendo la digitalización de su taller profesional.

\footnotetext{
${ }^{1}$ Para una descripción completa, $c f . \S 5.2 .3$ de MCER (2002, pp. 120-127).
} 
Esta perspectiva nos obliga a incluir el componente estratégico "compuesto por estrategias de comunicación verbal y no verbal que pueden ponerse en acción para compensar las fallas en la comunicación debido a variables de desempeño o a una competencia insuficiente" (Canale, Swain, 1980, p. 30) y aplicado, ante todo, a niveles de competencia bajos, y a los componentes gramatical y sociocultural. Para nuestro análisis es de suma importancia la falta de competencia que recoge la definición ${ }^{2}$ y que se encuentra plasmada en la capacidad de aprender del MCER (2002, pp. 104106). Esta competencia general incluye entre las destrezas del estudio, "la capacidad de utilizar materiales disponibles para el aprendizaje independiente" y "de organizar y utilizar materiales para el aprendizaje autodirigido".

Con el fin de conocer las necesidades, las expectativas, pero también los hábitos y las estrategias de los aprendientes, a lo largo del cuatrimestre de verano del curso académico 2018/2019 los estudiantes de Filología Española (FE) del Departamento de Filología Románica de la Universidad de Wroclaw fueron sometidos a una encuesta dedicada a la práctica de varias formas de expresión escrita (tanto en su lengua materna, como en otras lenguas que dominan) en el ámbito privado, académico y profesional. La metodología aplicada se basó en la perspectiva émica (Pike, 1967), que implica al estudiante en la reflexión sobre su propio proceso de aprendizaje.

Del análisis de los resultados se deduce que los estudiantes criticaron, tachando de inútiles, toda la serie de formas incluidas en los programas de la asignatura $P N J H$ Pisanie $^{3}$. En este estudio intentaremos diagnosticar las causas de tal valoración, tomando como punto de referencia por una parte, las competencias pragmáticas consideradas por los estudiantes como cruciales en su futura vida profesional y, por otra parte, las necesidades académicas reflejadas en los programas de estudios. La brecha entre estos dos grupos ha de revelar a qué estrategias recurren los estudiantes de FE a fin de salvarla.

\section{LA EXPRESIÓN ESCRITA COMO PARTE DEL PROGRAMA DE INSTRUCCIÓN LINGÜÍSTICA EN FILOLOGÍA ESPAÑOLA}

El programa de instrucción lingüística realizado en el Departamento de Filología Románica sigue las directrices de los documentos curriculares vigentes; sin embargo, y tal como se puede leer en un documento interno, presta a la destreza de la expresión escrita más atención que el MCER o el PCIC. La decisión se debe al perfil de estudiante que "a lo largo de sus estudios ha de tomar notas en una lengua extranjera, escribir exámenes y trabajos monográficos en este idioma y, por último, redactar en él

\footnotetext{
${ }^{2}$ Para profundizar en la evolución del tema de las estrategias, remitimos al lector a Cyr (1998).

${ }^{3}$ Las siglas en polaco PNJH (Praktyczna Nauka Języka Hiszpańskiego) Pisanie corresponden a Enseñanza práctica de la lengua española: Lengua escrita.
} 
su trabajo de fin de máster". No obstante, en dicho documento se pretende conciliar la perspectiva académica con la externa: a la expresión escrita se le dedica tanta atención también porque los empleadores "esperan que los graduados en FE sepan llevar la correspondencia o redactar textos de carácter funcional", hecho que se confirma en las encuestas realizadas a los graduados en Filología Románica, de cuyas carreras profesionales la Facultad realiza un seguimiento. Por eso, en los programas se pone énfasis en las "formas de expresión necesarias durante la formación académica (resúmenes, reseñas, textos argumentativos)", sin descuidar las practicadas en la vida profesional (informes, solicitudes, peticiones, quejas, reclamaciones).

Los estudiantes de primer ciclo, en función de su conocimiento previo de español, disponen de entre 660 y 780 horas de clases prácticas de instrucción lingüística, dedicando la cuarta parte de este tiempo a las clases de lengua escrita. En el segundo ciclo los estudiantes realizan 120 horas de un programa de instrucción lingüística configurado por Destrezas integradas, Español para fines profesionales, Estilística práctica de la lengua española y Redacción de textos académicos. Aunque solo el último curso está orientado exclusivamente a la destreza de expresión escrita en su variante académica, merece la pena observar que todos los demás cursos incluyen el perfeccionamiento del taller de redacción de textos. Además, los estudiantes que se especializan en Traducción realizan adicionalmente 30 horas del curso de Traducción literaria y los que optan por Filología digital, disponen de 30 horas de Escritura creativa (trabajo con el blog) ${ }^{4}$.

Como se puede apreciar, en el programa de ambos ciclos el desarrollo de la destreza de expresión escrita ocupa un lugar importante, ajustándose además, en el segundo ciclo, a los requisitos relacionados con la futura carrera profesional del estudiante. La importancia que se le atribuye se debe a su carácter transversal y a la convicción de que "la expresión escrita es una destreza difícil, sin embargo imprescindible para dominar una lengua extranjera. Es donde se funden al mismo tiempo [...] todas las destrezas con el conocimiento de subsistemas de la lengua, haciendo el proceso didáctico coherente y transparente. [...] La expresión escrita constituye una valiosísima mezcla de todas las operaciones lingüísticas" (Lipińska, 2016, p. 9).

\section{PERFIL DE LOS ENCUESTADOS}

En la encuesta participaron más de 80 estudiantes de ambos ciclos, la mayoría de ellos mujeres (80\%), de edades comprendidas entre 18 y 26 años. Es interesante observar que la carrera de FE no constituía su primera experiencia académica para un cuarto de los estudiantes de primer ciclo (la mayoría de ellos, con experiencia en

\footnotetext{
${ }^{4}$ Consúltense los programas de estudios en FE de primer ciclo: http://ifr.uni.wroc.pl/sites/default/files/ hl_plan_studiow_2019.pdfydesegundociclo:http://ifr.uni.wroc.pl/sites/default/files/filologia_hiszpanska_ ii.pdf.
} 
carreras no filológicas) y para la mitad de estudiantes de segundo ciclo (el 35\% del total con diploma en otra filología). Nos parece relevante resaltar este dato, ya que es de esperar que estos estudiantes se orienten mejor que los principiantes en las peculiaridades de los estudios universitarios, sabiendo comparar y contrastar el programa de estudios de FE con otras carreras conocidas. La mayoría de los estudiantes (más del $80 \%)$ tiene experiencia profesional: en gastronomía (27\%), trabajos de oficina (16\%), comercio $(15 \%)$ y educación $(12 \%)$.

Si nos fijamos en la presencia de la expresión escrita en la vida privada de nuestros estudiantes, constataremos que son representantes de la generación 160, es decir, para comunicarse rápida y eficazmente suelen recurrir al servicio de mensajes cortos de hasta 160 caracteres (SMS). Y, efectivamente, casi el 100\% de los estudiantes se dedica a la redacción de mensajes cortos: el $40 \%$ suele mandar entre 20 y 100 mensajes por día y el $20 \%$ sobrepasa este número, llegando hasta 1000 mensajes diarios. Casi todos los estudiantes (95\%) escriben correos electrónicos, eso sí, en cantidades mucho más reducidas, de costumbre, entre 1 y 2 por semana aunque hay quienes mandan hasta 20 mensajes por día. La condición del estudiante influye en la producción de notas y apuntes (tomados tanto durante las clases, como fuera de ellas): el $86 \%$ de los encuestados redacta entre 1 y 5 notas o apuntes por día. Más de la mitad $(60 \%)$ de los estudiantes mantiene la tradición de escribir postales, con un número medio de hasta 6 por año. Entre las formas que con menos frecuencia practican se encuentran: diarios $(16 \%)$, comentarios en blogs de otras personas (10\%) y entradas en su propio blog $(9 \%)$. Se observa una diferencia significativa en la producción de textos oficiales y formas de expresión artística entre el primer y segundo ciclo:

\begin{tabular}{|l|c|c|}
\hline \multicolumn{1}{|c|}{ Tipo de textos } & primer ciclo & segundo ciclo \\
\hline formales / oficiales & $14 \%$ & $37 \%$ \\
\hline de expresión artística & $24 \%$ & $47 \%$ \\
\hline
\end{tabular}

Los estudiantes de segundo ciclo suelen redactar más textos formales y de expresión artística. Entre los últimos predominan traducciones (de 2 por semana a 5 por año), poemas, cuentos (en algunos casos escritos con una enorme disciplina "2000 palabras 2 veces a la semana como mínimo, y entre 6000 y 10000 palabras cada día en períodos sin clases") y entrevistas. Lo que puede sorprender es que los estudiantes declaran falta de interés por compartir sus reflexiones en blog ( $3 / 4$ de los encuestados) o diario (más de $2 / 3$ ) y por comentar reflexiones en blogs ajenos (2/3); tampoco suelen ser muy activos en medios sociales (apenas 37\%). Eso contrasta, por ejemplo, con la constatación de que solo el 18\% de los estudiantes nunca escribe postales frente a un $82 \%$ que lo hace en algunas ocasiones. En este sentido, aunque los estudiantes de hoy son nativos digitales, suelen conservar bastantes hábitos tradicionales.

En cuanto al ámbito profesional, el 58\% de los encuestados declara realizar sus compromisos laborales sin recurrir a la expresión escrita. Si han de escribir algo, re- 
dactan: correos electrónicos $(28 \%)$, traducciones (16\%), presentaciones $(12 \%)$, encargos (12\%), informes (10\%) o esquemas de clases (10\%). En su futuro profesional esperan redactar correos electrónicos $(65 \%)$, traducciones $(62 \%)$, currículos $(43 \%)$, informes $(41 \%)$, presentaciones $(36 \%)$ y cartas oficiales $(28 \%)$. Los estudiantes subrayan que tanto en su experiencia laboral previa como en el futuro la lengua de sus estudios (español) aparece junto a otros idiomas: polaco, inglés u otras lenguas que dominan. Sorprendentemente, si comparamos las listas de formas practicadas o por practicar en la vida profesional con la lista de formas que, según los estudiantes, han de trabajarse a lo largo de sus estudios, veremos que no coinciden. El 65\% espera escribir correos electrónicos, pero solo el 19\% quiere que se enseñe esta forma en clase y, si le concede un hueco en el programa, es solo para ver "qué cosas evitar y qué errores se cometen con mayor frecuencia". Por otra parte, aunque solo el $28 \%$ espera redactar cartas oficiales, hasta el $80 \%$ desea trabajar esta forma en clases. Solo un $13 \%$ quiere aprender a hacer traducciones frente a un $31 \%$ que desea trabajar en clase con formas de expresión artística, ya que "fomentan el desarrollo personal, desarrollan la creatividad y abren a otras personas".

\section{RESULTADOS Y ANÁLISIS}

\subsection{EL PROGRAMA DE PNJH PISANIE}

La mayoría de los estudiantes (3/4) considera que la carga horaria de las clases de expresión escrita es suficiente para realizar el programa y conseguir los objetivos que se marcan. Se puede apreciar que los estudiantes complementan el trabajo realizado en clase dedicando a la redacción de trabajos escritos de todo tipo entre 1 y 10 horas por semana. Es interesante observar que los estudiantes a los que les gustaría disponer de más horas de clases de redacción de textos vienen sobre todo de segundo ciclo (29\%), los de primero mayoritariamente se dan por satisfechos con las horas ofrecidas en el programa (83\%).

Según las encuestas, los estudiantes consideran que el tiempo dedicado a la práctica de redacción de textos se podría aprovechar mejor: hasta $2 / 3$ de los encuestados cree que no todas las formas practicadas en clase son necesarias. Dentro de las innecesarias destacan cinco grupos: a) formas que suelen practicar en su vida privada (aunque muchas veces parecen olvidarse de que tener competencia en la expresión escrita en la L1 no se traduce en poseerla en la LE; Giovannini et al., 2000, p. 79); b) formas que no usan en su lengua materna (diarios, blogs, comentarios); c) formas breves, como postales o invitaciones, consideradas triviales ("seamos sinceros, que no es ingeniería aeroespacial"); d) formas de expresión artística ("escribir relatos cortos es muy fácil y no requiere comentarios", "relatos y cuentos no son precisamente formas que me serán necesarias en la vida cotidiana en el futuro", además "si a alguien le 
interesa, puede trabajarlas por su cuenta"); y e) formas que vuelven en varias etapas de formación, como, por ejemplo, los currículos (aunque casi la mitad cree necesitarlos en su trabajo). Es llamativo que los estudiantes de segundo ciclo son mucho más cautelosos a la hora de calificar de inútil cualquier forma o género.

\subsection{COMPETENCIAS PRAGMÁTICAS}

¿Qué obstaculiza, según los encuestados, el buen funcionamiento de dicha combinación? ¿Qué elementos requieren más atención? Lo que más les preocupa a los estudiantes son las deficiencias de su competencia lingüística: consideran que necesitan dedicarse más al estudio de construcciones gramaticales, trabajar la sinonimia -tanto léxica, como sintáctica- y mejorar su dominio de las normas ortográficas. Les interesaría también ampliar su competencia sociolingüística: conocer más coloquialismos y aprender a escoger el estilo (formal frente a informal) en función del tipo de documento, destinatario y tema tratado.

Conforme crece el nivel de dominio de la lengua, aumenta la conciencia de que el conocimiento del vocabulario y de las estructuras gramaticales es insuficiente para una comunicación eficiente y correcta. Son, sobre todo, los estudiantes de segundo ciclo los que formulan postulados relacionados con las competencias pragmáticas. Intuyen que, sin estas, los textos carecerán de organización, estructura y orden, no permitirán identificar la función que han de cumplir ni seguir la relación de causa-efecto, no respetarán normas de interacción y, como resultado, presentarán problemas de cohesión o de coherencia. Por eso, en el programa de la asignatura de PNJH Pisanie se presta mucha atención a los fenómenos transfrásticos, tipos de progresión temática, problemas de cohesión interna, etc. Los encuestados parecen compartir este punto de vista, reclamando que el desarrollo de la destreza de expresión escrita abarque la "corrección pragmática", identificando como sus componentes la "cohesión" conseguida sobre todo gracias al "uso correcto de nexos", la "capacidad de darle al texto una estructura adecuada" relacionada con el empleo de "expresiones típicas de un género textual". Los estudiantes quieren mejorar su "capacidad de formular ideas de forma clara", "formular con fluidez tesis y argumentos claros" a fin de lograr que "el texto sea coherente y lógico al mismo tiempo que interesante para el destinatario".

\subsection{AUTOAPRENDIZAJE Y COMPETENCIAS ESTRATÉGICAS}

Los encuestados subrayan la importancia del desarrollo de la competencia estratégica y del saber aprender, reclamando que se les inculque "una postura creativa a una posible falta de competencias, desconocimiento de palabras o expresiones". Por supuesto, la carga horaria no permite que dichas competencias se desarrollen ple- 
namente en clase bajo la tutela de un profesor, por eso el programa incluye ciertos procedimientos basados en el aprendizaje inductivo (por ejemplo, el análisis de textos, algunas veces reforzado por el análisis contrastivo a fin de evitar interferencias de la lengua materna) que el aprendiente ha de continuar en forma de aprendizaje autónomo. Las encuestas demuestran que la mayor parte de nuestros estudiantes (casi 2/3) intenta poner en práctica estos procedimientos invirtiendo tiempo y fuerzas, aunque, según veremos, algunos lo hacen un poco a ciegas.

Entre los procedimientos utilizados por los encuestados en su tiempo libre para reforzar el trabajo de clase predomina la lectura. Esta indicación no debe sorprender, ya que estas dos destrezas están fuertemente unidas y la expresión escrita no puede existir si no la acompaña la lectura. Sin embargo, es crucial saber qué leen los encuestados, cómo y para qué lo hacen.

La mayoría de los estudiantes enumera entre sus lecturas simplemente "textos en español"; sin embargo, de otros comentarios se deduce que estos textos provienen principalmente de la prensa, de los blogs redactados por usuarios nativos y de varias obras literarias. Es una selección un tanto sorprendente, ya que en todas las encuestas los estudiantes dejan claro que ni escriben blogs, ni piensan hacerlo, ni lo consideran útil. Tampoco los géneros periodísticos se encuentran en la lista de formas que deberían enseñarse en el programa de estudios y, tal como hemos mencionado, las formas de expresión artística no las consideran "precisamente formas que [...] serán necesarias en la vida cotidiana en el futuro". Se puede suponer que los encuestados indican justamente estas formas como primera opción a causa de la motivación (lo que leen les interesa y entretiene) o porque es donde pretenden encontrar aquella riqueza léxica y estructural que buscan. Los blogs, por su parte, probablemente suplen deficiencias de estilo coloquial que los estudiantes señalan. Pocas personas indican como fuente de textos leídos los textos ejemplares o canónicos, que probablemente abarcan modelos de diferentes formas escritas. Cuando leen algo, los encuestados no se limitan a una lectura pasiva: "leo atentamente, prestando atención a la estructura del texto y analizando qué podría aprovechar" o "leyendo textos, voy subrayando expresiones que me pueden servir".

Varios encuestados indican también que aprenden a escribir escribiendo, algunos de ellos sin darse cuenta de que lo que practican tiene pocas características propias de la lengua escrita: "chateo con mis amigos españoles". Otros "intentan escribir de forma individual, haciendo apuntes" o "escriben artículos y cuentos por su propia iniciativa". Es alentador observar que los encuestados se preocupan por la corrección, recurriendo a la ayuda de otras personas ("a veces escribo varios textos y los dejo a mis amigos o a otras personas calificadas para que los corrijan", "publico cuentos o traducciones en Internet lo que me ayuda mucho, pues por lo general la gente, si ve algún error, lo señala"), o sometiendo su propia producción a la autocorrección ("escribo mucho, corrijo mis propios errores y vuelvo a leer").

Algunos de los encuestados creen practicar la expresión escrita haciendo traducciones e "intentan traducir todo lo que encuentren por su camino" o "publicando sus 
traducciones en Internet”. La mayoría, sin embargo, trabaja sobre todo el vocabulario: "buscando sinónimos" o "expresiones en diccionarios bilingües", o consultando sus dudas con los profesores. Muchos estudiantes admiten también hacer ejercicios de gramática.

En su trabajo autónomo los estudiantes recurren ante todo a Internet (2/3), diccionarios (54\%) o manuales y métodos (45\%). Es sorprendente que ninguno de los encuestados haya indicado corpus lingüísticos ni herramientas informáticas de traducción, aunque son herramientas con las que se les familiariza durante las clases.

\section{CONCLUSIONES}

Los estudiantes de FE siguen escribiendo relativamente mucho y usando formas variadas. Aunque emplean las nuevas tecnologías que facilitan especialmente la interacción escrita (mensajes de texto, correos electrónicos), no han abandonado del todo los medios tradicionales (postales); si desean expresar lo que sienten, pocas veces lo escriben en blogs o foros, pero, sí, con bastante frecuencia, dan a sus vivencias forma artística.

El programa de estudios que les ofrecemos cumple parcialmente con sus expectativas sobre la destreza de expresión escrita, también desde el punto de vista pragmático, garantizando un espacio adecuado para compaginar el trabajo guiado (realizado en clase) con el individual. Las formas enseñadas no siempre coinciden con las necesidades y los intereses de los encuestados, aunque se puede observar que los de segundo ciclo aprecian mucho más las numerosas formas consideradas inútiles por sus compañeros de primer ciclo. Quizás este hecho se debe a que dichos estudiantes, gracias a una experiencia laboral (y, ante todo, al desempeño de cargos relacionados con su formación) que requiere unos usos específicos de la lengua, han descubierto que no todas las formas consideradas como triviales realmente lo son.

Las encuestas parecen confirmar que la fecha de nacimiento no necesariamente se traduce en competencias digitales: por una parte, no se puede suponer que los estudiantes entran en la universidad dotados de ciertos conocimientos y habilidades; hay que mostrarles las posibilidades y los riesgos que conllevan; por otra parte, las competencias junto con las herramientas que ayudan a conseguirlas no pueden ser ignoradas, puesto que el mundo laboral se las va a reclamar.

Parece que una de las competencias más valiosas que la carrera filológica puede ofrecer al estudiante es la competencia estratégica y su tipificación en el Marco: "saber aprender", la cual, junto con el hábito de trabajo autónomo, le ayudará a solventar cualquier problema. Para que el autoaprendizaje sea más efectivo, se debe fomentar la inteligencia intrapersonal y la capacidad de reflexión, en general. Por supuesto, aunque los estudiantes de FE demuestran que son capaces de desarrollar competencias pragmáticas con gran dosis de autonomía, los programas universitarios 
deben acompañar la evolución experimentada por el taller de trabajo y comunicación intercultural en los nuevos medios. Esta, a su vez, requiere disponer de tres grupos de habilidades: técnicas (en lo que se refiere al manejo del canal de comunicación), escribir rápido con el teclado y ser consciente de las exigencias del entorno comunicativo

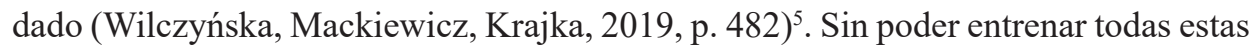
destrezas, de lo que sí somos capaces es de sensibilizar a los futuros filólogos sobre cierto tipo de metapragmática, que no se limita al conocimiento de esquemas sino que permite extraerlos gracias al análisis, puesto que las funciones discursivas y dichos esquemas también están en evolución constante. Empujando a los estudiantes hacia la autonomía, despertando su curiosidad cognitiva, enseñándoles a gestionar la diversidad y permitiéndoles salir de su aprendida indefensión, los preparamos mejor para enfrentarse al mundo que les espera fuera de los muros de su Alma Mater.

${ }^{5}$ Los autores sostienen que "durante las interacciones llevadas a cabo en Internet la competencia sociolingüística, crucial en la comunicación directa, desempeña un papel menos destacado". 


\section{BIBLIOGRAFÍA}

Canale, M., Swain, M. (1980). Theoretical Bases of Communicative Approaches to Second Language Teaching and Testing. Applied Linguistics, 1, 1-47.

Consejo de Europa (2002). Marco común europeo de referencia para las lenguas: aprendizaje, enseñanza, evaluación. Madrid: Ministerio de Educación, Cultura y Deporte.

Cyr, P. (1998). Les stratégies d'apprentissage. Paris: CLE International.

Giovannini, A., Martín Peris, E., Rodríguez Castilla, M., Simón Blanco, T. (2000). Profesor en acción 3. Destrezas. Madrid: Edelsa.

Harari, Y.N. (2018). 21 lekcji na XXI wiek (M. Romanek, Trans.). Kraków: Wydawnictwo Literackie (Original work published 2018).

Hulin, T., Pélissier, Ch. (2012). Appropriation de l'écriture numérique: évaluation et parcours pédagogique. In Fondements théoriques, représentations, réalités de l'expression-communication dans les IUT - Bilan et perspectives (pp. 1-15). Recuperado de: https://halshs.archives-ouvertes.fr/ halshs-00786651/document.

Lipińska, E. (2016). Pisanie - sprawność niezbędna w kształceniu językowym. Języki Obce w Szkole, 2, 9-14.

Pike, K. (1967). Language in Relation to a Unified Theory of Structure of Human Behavior. La Haye: Mouton.

Prensky, M. (2001). Digital Natives, Digital Immigrants. On the Horizon, 9 (5), 1-6. DOI: 10.1108/ 10748120110424816.

Wilczyńska, W., Mackiewicz, M., Krajka, J. (2019). Komunikacja interkulturowa. Wprowadzenie. Warszawa: PWN. 